\title{
BICEP: a cosmic microwave background polarization telescope at the South Pole
}

\author{
Yuki D. Takahashi for the BICEP collaboration \\ Department of Physics, University of California, Berkeley, CA 94720-7300, USA \\ email: yuki@bolo.berkeley.edu
}

\begin{abstract}
BICEP was a telescope designed to probe the polarization of the cosmic microwave background $(\mathrm{CMB})$ for the signature of gravitational waves produced during the epoch of inflation. The instrument was developed by a team of scientists from Caltech/JPL, UC Berkeley, and UC San Diego. It was installed at the South Pole in November 2005 and the CMB observations were conducted from February to November each year with one winter-over scientist responsible for operating and maintaining the instrument. Taking advantage of the excellent atmospheric conditions at the South Pole, we mapped $2 \%$ of the sky at 100 and $150 \mathrm{GHz}$. We completed 3 years of observations from 2006 to 2008, mapping the CMB polarization anisotropy at degree angular scales with unprecedented sensitivity. In 2010, a next generation instrument, BICEP2, will be installed on the existing telescope mount for an even deeper survey.
\end{abstract}

Keywords. Cosmic Microwave Background, Instrumentation: polarimeters, Gravitational Waves.

\section{Introduction}

BICEP was specifically designed to search for a signature of gravitational waves from inflation by studying the polarization of the CMB. The ultimate goal is to find direct evidence for inflation through gravitational waves that would have been generated during inflation. Those gravitational waves would have resulted in polarization of the CMB with a "B-mode" pattern. The resulting B-mode polarization anisotropy has an angular power spectrum that is expected to peak at around $2^{\circ}$ angular scales, and whose magnitude allows us to constrain the inflationary energy scale.

\section{Experiment Design}

Because the potential signal is expected to be under a $\mu \mathrm{K} \mathrm{rms}$, the design priorities for BICEP were sensitivity and systematic error control. For maximum sensitivity, we chose the South Pole site because of its highly transparent and stable atmosphere. We observed in atmospheric transmission windows near the peak of the CMB blackbody spectrum, at two frequency bands (100 and $150 \mathrm{GHz}$ ) as a guard against potential foreground contamination.

The BICEP telescope has a modest aperture of $25 \mathrm{~cm}$, leading to a beam size of $\sim 1^{\circ}$, which is adequate to resolve the B-mode anisotropy at its peak. This small aperture allows for aggressive shielding of sidelobes as well as simple and effective implementation of calibration measurements. To measure CMB polarization, we difference pairs of polarization sensitive bolometers (PSBs), similar to those recently launched on the Planck space-based telescope. The telescope is a simple on-axis refractor with feedhorns coupling the radiation onto 49 pairs of PSBs. 


\section{Deployment and Observations}

We integrated and tested the instrument from 2003 to 2005 and deployed it to the South Pole in November 2005 for 3 years of operation until December 2008. During the summer seasons, we performed careful calibration measurements to characterize the instrument and to ensure that systematic errors are subdominant to the noise level. Takahashi et al. (2009) describes the instrumental properties characterized, including the bolometer temporal response, PSB pair beam mismatch, far sidelobes, spectral bandpass, polarization orientations and polarization efficiency. For CMB observations, we chose the cleanest available $2 \%$ of sky where the dust emission is minimized at $150 \mathrm{GHz}$. We also observed polarized emission from the Galaxy, including that from mid-latitude dust.

\section{Results}

BICEP achieved mapping depth on the Galaxy similar to that expected by the Planck survey. Our maps of polarized emission from the Galactic plane are being used to calibrate the polarized response of Planck. In our CMB field, BICEP probes much deeper. Our Emode spectrum is sample variance limited up to the first peak. BICEP has lowered the upper limits on the B-mode by an order of magnitude with only the first 2 years of data and aggressive cuts. From the analysis of these data, the tensor-to-scalar ratio has been constrained to $r<0.73$ with $95 \%$ confidence (Chiang et al. 2009). Systematic errors are controlled to well below this limit, and the analysis of the full 3-year data is ongoing.

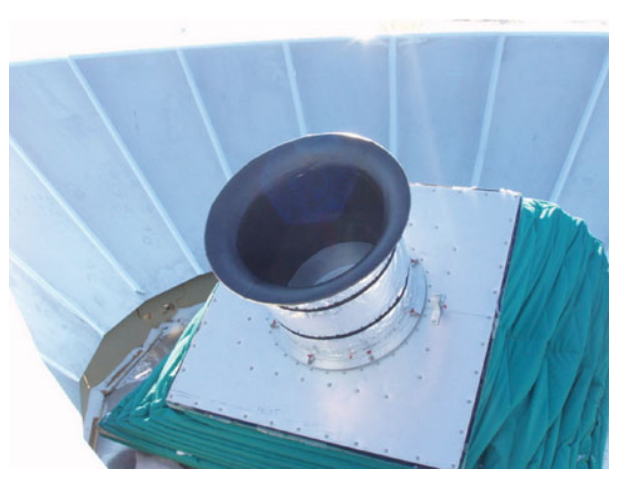

Figure 1. BICEP at the South Pole.

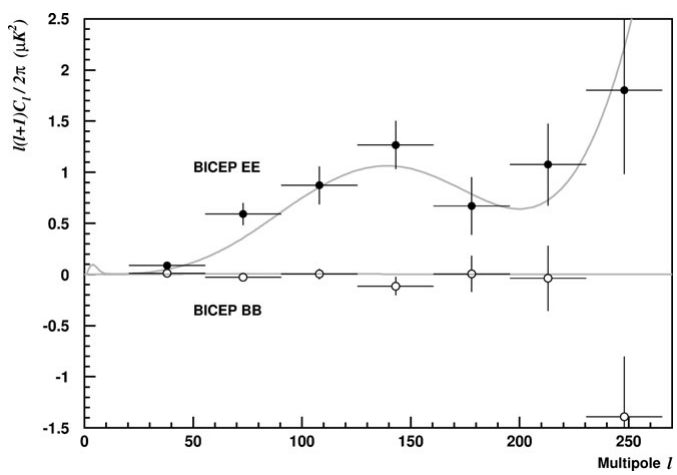

Figure 2. Two-year polarization spectra.

\section{Future}

Observations will continue in 2010 with BICEP2, which has a highly packed antennacoupled transition-edge sensor bolometer array. With over 200 PSB pairs, mapping speed is expected to increase $\sim 5$ fold. Extrapolating from the achieved sensitivity of BICEP, BICEP2 can expect to reach the sensitivity necessary to begin probing physically interesting ranges of amplitudes for gravitational wave signal from inflation.

We acknowledge support by NSF Grant OPP-0230438 and the US Antarctic Program.

\section{References}

Chiang, H. C., et al. 2009, submitted to ApJ, arXiv:0906.1181

Takahashi, Y. D., et al. 2009, submitted to ApJ, arXiv:0906.4069 